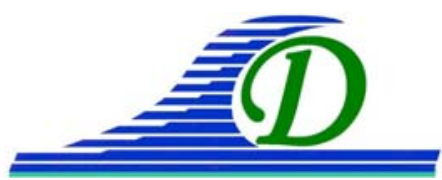

XIII ${ }^{\text {èmes }}$ Journées Nationales Génie Côtier - Génie Civil

Dunkerque, 2-4 juillet 2014

DOI:10.5150/jngcgc.2014.033 C Editions Paralia CFL

disponible en ligne - http://www.paralia.fr - available online

\title{
Etude hydro-sédimentaire de la Rade de Toulon
}

\author{
Christiane DUFRESNE ${ }^{1,2}$, Céline DUFFA ${ }^{1}$, Vincent REY ${ }^{2}$
}

1. Institut de Radioprotection et Sûreté Nucléaire, PRP-ENV/SESURE/LERCM, Zone portuaire Brégaillon - Centre Ifremer, CS20330, 83507 La Seyne sur Mer, France.

2. Aix Marseille Université, CNRS/INSU, IRD, Mediterranean Institute of Oceanography (MIO), UM 110, 13288 Marseille / Université de Toulon, CNRS/INSU, IRD, Mediterranean Institute of Oceanography (MIO), UM 110, 83957 La Garde, France.

christiane.dufresne@irsn.fr ; celine.duffa@irsn.fr ; rey@univ-tln.fr

\section{Résumé :}

L'accident nucléaire de Fukushima de mars 2011 a montré l'importance de pouvoir simuler le devenir des radionucléides en milieu marin et de disposer d'un outil de gestion post-accidentelle. Ainsi, l'Institut de Radioprotection et de Sureté Nucléaire (IRSN) met en place un modèle hydro-sédimentaire de la Rade de Toulon, port d'attache de bâtiments à propulsion nucléaire. En plus de leur décroissance radioactive, le devenir des radionucléides en mer est conditionné par de nombreux processus physiques, tels l'hydrodynamisme et les processus sédimentaires. Puisque certains radionucléides présentent une affinité particulière pour la phase particulaire, les sédiments jouent un rôle de source ou de puits de contamination par la remise en suspension et le dépôt. La précision du modèle hydro-sédimentaire sera ainsi déterminante sur la prévision du devenir de la contamination à moyen et long terme. Les premiers résultats montrent une bonne représentation de la circulation de la Rade par le modèle ainsi que des échanges entre ses deux bassins (Grande Rade et Petite Rade), fortement corrélés aux épisodes ventés. Dans le but d’obtenir des simulations réalistes, les apports des cours d'eau se jetant dans la Rade sont pris en compte dans le modèle. Afin d'étudier ces apports, des pièges à sédiments et sondes multi-paramètres ont été installés dans ces deux cours d'eau, le Las et l'Eygoutier. De plus, des courantomètres, altimètres et turbidimètres ont été mouillés en rade à proximité des exutoires dans le but d'étudier la dynamique sédimentaire en mer. Les données de turbidité et altimétriques révèlent diverses périodes de dépôt et d'érosion lors d’évènements météorologiques marqués. Dispersés dans un panache en mer, les apports des fleuves contiennent principalement des particules fines. Les simulations montrent l'évolution du panache aux exutoires et permettent d'estimer le devenir sédimentaire en mer.

Mots-clés : Modélisation, Hydrodynamisme, Dynamique sédimentaire, Apports des bassins versants, Rade de Toulon. 


\section{Thème 2 - Dynamique sédimentaire}

\section{Introduction}

L'accident nucléaire de Fukushima de mars 2011 a montré l'importance de pouvoir simuler le devenir des radionucléides en milieu marin et de disposer d'un outil de gestion post-accidentelle. Ainsi, l'Institut de Radioprotection et de Sureté Nucléaire (IRSN) met en place un modèle hydro-sédimentaire de la Rade de Toulon, port d'attache de bâtiments à propulsion nucléaire. En plus de leur décroissance radioactive, le devenir des radionucléides en mer est conditionné par de nombreux processus physiques, tels l'hydrodynamisme et les processus sédimentaires (MONTE et al., 2009). Le transport par les courants et la dispersion due à la turbulence auront un impact sur le devenir de la contamination. Puisque certains radionucléides présentent une affinité particulière pour la phase particulaire, les sédiments jouent un rôle de source ou de puits de contamination par la remise en suspension et le dépôt et de dispersion à travers leur transport, en particulier en suspension. La précision du modèle hydro-sédimentaire sera donc déterminante sur la prévision du devenir de la contamination. Le travail présenté ici vise donc à améliorer les connaissances concernant la dynamique sédimentaire dans la Rade de Toulon pour une bonne prise en compte des radionucléides adsorbés à la surface des sédiments. Cette étape est essentielle au développement d'un futur outil de prévision de la dispersion de radionucléides en cas de rejet accidentel.

La Rade de Toulon est une baie enclavée, séparée en deux bassins (Grande Rade et Petite Rade) par une digue longue de 1,4 km. La Petite Rade située dans la partie ouest est quasi fermée : elle est reliée à la Grande Rade par une petite passe (d'environ $30 \mathrm{~m}$ de large et $10 \mathrm{~m}$ de profondeur) et une grande passe au sud, aussi appelé chenal au vue de la navigation (figure 1). Cette dernière, d'une largeur d'environ $1 \mathrm{~km}$ et d'une profondeur maximale de $30 \mathrm{~m}$, est le principal lieu d'échange entre les deux bassins. La zone étudiée est entourée par un relief accidenté dont les massifs canalisent la direction des vents et jouent un rôle sur les précipitations. La région est caractérisée par un climat méditerranéen, c'est-à-dire un fort ensoleillement et de courtes périodes de pluies, dont les évènements sont souvent brefs mais intenses. Il y règne deux types de vent distincts : le Mistral (nord-ouest) et le Vent d'Est (est - nord-est) soufflant tous deux environ 40\% du temps (JOUAN et al., 2001). Les conditions météorologiques ont un impact direct sur la circulation dans la Rade de Toulon, les vents influençant directement son hydrodynamisme (MILLOT et al., 1981 ; DUFRESNE et al., 2014). La granulométrie du fond sédimentaire varie par endroits, mais contient principalement des vases, notamment dans la Petite Rade où les particules fines sont prédominantes dans la couche de surface (figure 2) (TESSIER et al., 2011 ; ARNAUD \& CHARMASSON, 2000). La proportion de sable augmente considérablement à proximité des plages artificielles sur la côte nord de la Grande Rade ainsi que dans la partie sud du domaine, où elle atteint jusqu'à $90 \%$. Le type de sédiment aura une part importante dans la modélisation de la dispersion des radionucléides. En effet, les sédiments fins, plus 


\section{XIII ${ }^{\text {èmes }}$ Journées Nationales Génie Côtier - Génie Civil \\ Dunkerque, 2-4 juillet 2014}

facilement mobilisables, ont aussi la propriété de piéger davantage les contaminants, au vu de leurs propriétés physico-chimiques.

Les deux principaux fleuves côtiers se jetant dans la Rade de Toulon sont ici étudiés. Tantôt naturels, tantôt urbanisés, ils traversent différentes zones résidentielles, agricoles ou commerciales. Le Las (8 km) et l'Eygoutier (15 km) s'écoulent respectivement dans la Petite et la Grande Rade et leurs débits sont corrélés aux épisodes pluvieux.

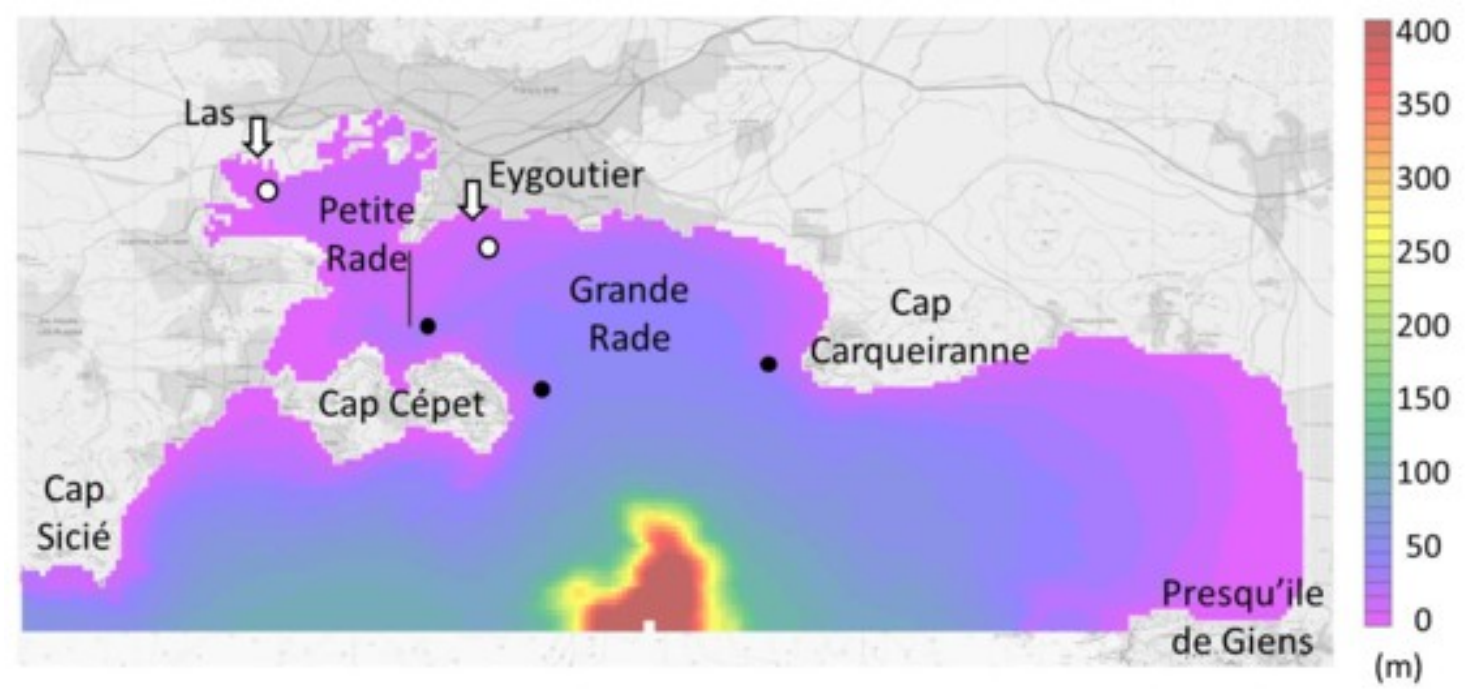

Figure 1. Bathymétrie (m) du domaine numérique modélisé et positions des instruments de mesure (cercles) et exutoires des fleuves (flèches).

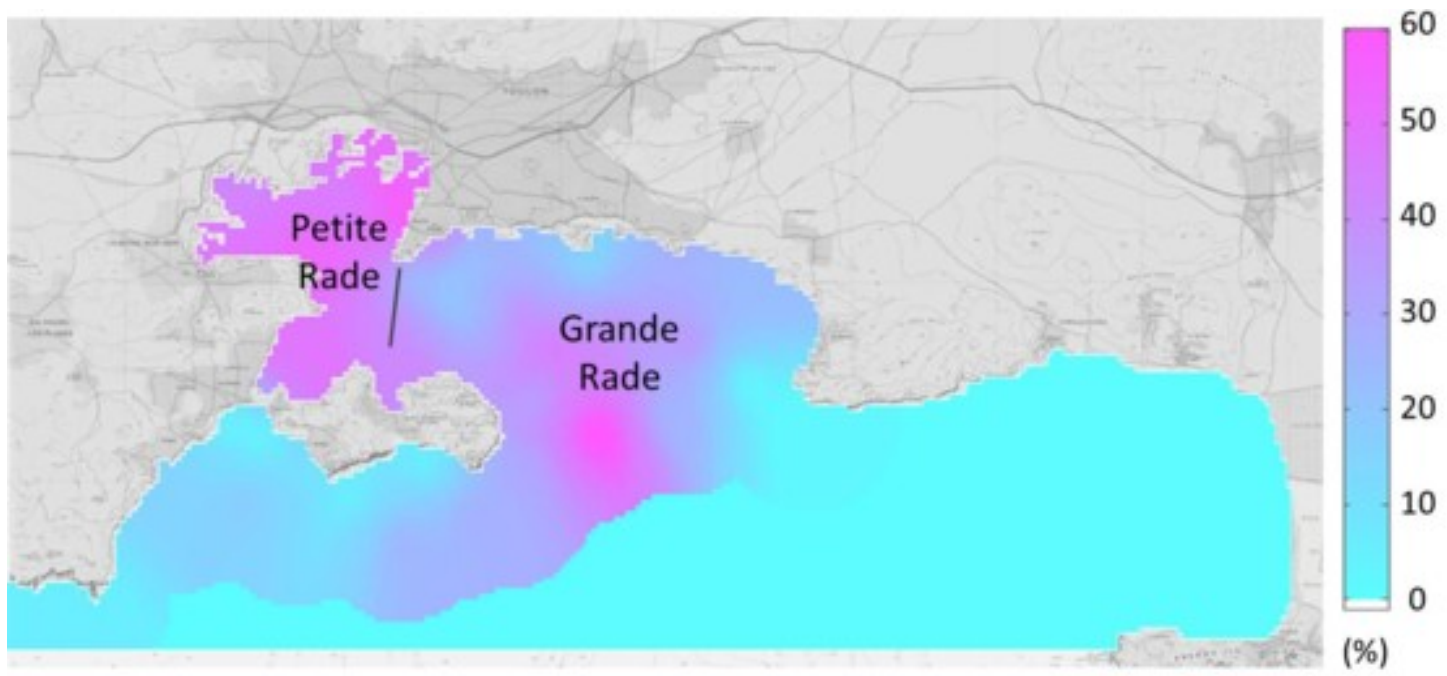

Figure 2. Carte granulométrique représentant la fraction (\%) de vase (diamètre $d<63 \mu \mathrm{m}) d u$ fond sédimentaire (extrapolation des données issues de ARNAUD \& CHARMASSON, 2000). 


\section{Méthodologie}

\subsection{L'instrumentation des cours d'eau}

Dans le but d'estimer les apports solides des fleuves à la Rade, une sonde multiparamètre et un piège à sédiments ont été mouillés dans chacun des cours d'eau pendant 18 mois. Ces sondes autonomes mesurent la température, la pression, la conductivité électrique et la turbidité. Les données enregistrées sont post-traitées afin de filtrer les éventuelles mesures aberrantes. Les mesures de pression atmosphérique sont dans un premier temps retranchées de façon à ce que la pression mesurée concerne uniquement la colonne d'eau et les données hors d'eau (conductivité électrique nulle) sont éliminées. Enfin, les enregistrements de turbidité sont filtrés : lorsqu'une donnée présente des écarts absolus supérieurs à 50 NTU entre sa valeur et la précédente ainsi qu'entre sa valeur et la suivante, elle est remplacée par la moyenne des valeurs des données l'encadrant. Les pièges à sédiments permettent d'échantillonner de façon intégrée dans le temps les matières en suspension (MES) dans un cours d'eau. Développé par PHILLIPS et al. (1999), le piège est placé dans le sens de l'écoulement et capture par décantation la MES. Le contenu du piège est ensuite évaporé et pesé, puis analysé au granulomètre laser. Des prélèvements ponctuels ont également été réalisés afin de valider les enregistrements de la sonde. D’abord tamisés à $2 \mathrm{~mm}$, ces échantillons sont filtrés à $45 \mu \mathrm{m}$, séchés à l'étuve puis pesés.

\subsection{L'instrumentation en mer}

Pour l'étude de l'hydrodynamisme et de la circulation dans la Rade, des courantomètres ont été mouillés en différentes positions (cercles noirs sur la figure 1). A l'exutoire des deux fleuves, différents instruments de mesure ont été mouillés afin d'étudier le devenir en mer des apports sédimentaires fluviaux. Pour ces sites (cercles blancs sur la figure 1), un courantomètre ADCPs Workhorse Sentinel (RDInstrument), un altimètre NKE-Altus et une sonde de turbidité WETLAB ont été mouillés par 10 et 20 m de profondeur pour le Las et l'Eygoutier respectivement.

\subsection{La modélisation hydro-sédimentaire}

Le modèle hydrodynamique tridimensionnel utilise le code MARS3D, fondé sur la résolution par différences finies des équations primitives de l’hydrodynamique, tel que décrit par LAZURE et DUMAS (2008). La configuration de la Rade de Toulon, mise en place et validée par DUFFA et al. (2011) et DUFRESNE et al. (2014), est définie par des mailles horizontales de $100 \mathrm{~m}$ et 30 niveaux sigma verticaux. L'emprise s'étend du Cap Sicié à l'ouest, à la Presqu'île de Giens à l'Est (figure 1). Les conditions de frontière ouverte (limite sud du domaine) sont imposées par un modèle de plus grande emprise couvrant la Méditerranée Nord-Occidentale (MENOR). Le modèle atmosphérique américain Mesoscale Model 5 (MM5) (http://www.ncar.ucar.edu) affiné 


\section{XIII ${ }^{\text {èmes }}$ Journées Nationales Génie Côtier - Génie Civil \\ Dunkerque, 2-4 juillet 2014}

à une résolution $3 \mathrm{~km}$ est utilisé comme forçage pour la surface libre (vent, pression, rayonnement). Les contraintes de cisaillement au fond associées à la houle sont calculées à partir du modèle de propagation SWAN. Les effets des vagues sont principalement notables dans la partie la plus exposée de la Grande Rade. La tension au fond prise en compte pour le modèle sédimentaire est la somme de la contrainte due au courant et celle due aux vagues.

Le modèle sédimentaire, détaillé par LE HIR et al. (2011), simule la dynamique sédimentaire en calculant les flux de concentrations de sédiment en suspension et de transport dans la colonne d'eau selon les forçages hydrodynamiques. Les sédiments sont classés selon leur taille, leur comportement cohésif et leur mode de transport. Pour la configuration de Toulon, le modèle utilise deux classes de sédiments : les sables, noncohésifs et transportés en suspension ou par saltation au fond, et les vases, particules cohésives transportées en suspension. Les flux d'érosion sont calculés en fonction du type de sédiments si la tension au fond $\tau$ est supérieure à $\tau_{i}$ la tension critique d'érosion, selon une loi érosive telle :

$E_{i}=E_{0}\left(\frac{\tau}{\tau_{i}}-1\right)^{n_{i}}$

avec $E_{i}$ l'érosion du sédiment, $E_{0}$ un paramètre érosif et $n_{i}$ un coefficient qui dépendent du type de sédiment $i$. Le flux de dépôt $D_{i}$ pour chaque classe $i$ est calculé selon la loi de Krone, avec $C_{i}$ la concentration de sédiment en suspension, $W_{s i}$ la vitesse de chute et $\tau_{d i}$ tension critique de dépôt :

$D_{i}=W_{s i} \cdot C_{i}\left(1-\frac{\tau}{\tau_{d i}}\right)$

Le transport en suspension est simulé par la résolution d'une équation d'advectiondiffusion pour la concentration des différentes classes de particules, suivant l'équation :

$\frac{\partial C_{i}}{\partial t}+\vec{U} \cdot g r \vec{a} d C_{i}+W_{s i} \frac{\partial C_{i}}{\partial z}=\frac{\partial}{\partial z} K_{z} \frac{\partial C_{i}}{\partial z}$

avec $C_{i}$ la concentration dans la colonne d'eau à proximité du fond, $W_{s i}$ la vitesse de chute et $K_{z}$ le coefficient de diffusion turbulente verticale.

\section{Résultats}

\subsection{Hydrodynamisme de la Rade}

L'étude des enregistrements des courantomètres montrent une préférence bidirectionnelle des courants sur l'ensemble de la Rade. Fortement influencés par les conditions météorologiques, les courants de surface sont entrainés par le vent et leur intensité est d'autant plus forte que les épisodes ventés sont prononcés. Le Mistral (nord - nord-ouest) entraine ainsi l'eau de surface vers l'extérieur de la Rade alors que le vent d'Est (est - nord-est) entraine le dessus de la colonne d'eau dans le bassin. Par endroit, 


\section{Thème 2 - Dynamique sédimentaire}

toute la colonne d'eau se dirige dans la même direction ; ailleurs, notamment dans le chenal de navigation, une inversion entre surface et fond est marquée entre 5 et $10 \mathrm{~m}$ de profondeur. Les courants de fond sont alors de direction opposée aux courants de surface et suivent la bathymétrie. Après un fort coup de Mistral, une renverse de courant de forte intensité est généralement constatée, sans dépendance significative vis à vis des conditions météorologiques (calmes ou ventées). Lors de changements de conditions atmosphériques, les courants s'inversent rapidement.

Les comparaisons modèle-mesures ont montré une bonne capacité du modèle à reproduire la circulation dans la Rade. Bien que légèrement sous-estimés au fond, les intensités de courants simulées correspondent à celles mesurées. Les renverses et inversions surface/fond sont également bien représentées.

De plus, les mesures en mer montrent des variations de température avec les épisodes de vent. Ainsi, le Mistral, favorisant les upwelling, engendre une diminution de la température par remontée des eaux de fond alors que le vent d'Est entraine un réchauffement des eaux par l'apport d'eau plus chaude du large (cf. épisodes postMistral 11, 15, 20 novembre 2013 de la figure 3d).

\subsection{Apports sédimentaires des fleuves}

Les instruments placés dans les cours d'eau ont permis dans un premier temps d'étudier les apports des fleuves. Très réactifs aux conditions météorologiques, le niveau d'eau d'étiage est plutôt faible et constant tout au long de l'année. En période de pluie, il augmente considérablement et de façon très dépendante de la pluviométrie. Les débits peuvent ainsi s'accroitre très rapidement. Les épisodes pluvieux se caractérisent aussi par une augmentation de la turbidité et une diminution de la conductivité électrique mesurées dans les fleuves, principalement due aux apports d'eau de pluie (cf. épisodes de pluie 18, 19, 21 novembre 2013 de la figure 3b,c). Par temps sec, la concentration de MES est en moyenne de $5 \mathrm{mg} \cdot \mathrm{L}^{-1}$ pour les deux fleuves et peut atteindre $1000 \mathrm{mg} \cdot \mathrm{L}^{-1}$ lors de fortes crues. Les sédiments prélevés dans les pièges montrent également une variation de masse totale en fonction de la pluviométrie. Les saisons automnale et hivernale, les plus pluvieuses, correspondent à des prélèvements par pièges plus importants. L'analyse granulométrique des sédiments prélevés révèle une très grande proportion ( $>90 \%$ ) de particules fines (diamètre $\mathrm{d}<63 \mu \mathrm{m}$ ). 
a)

b)

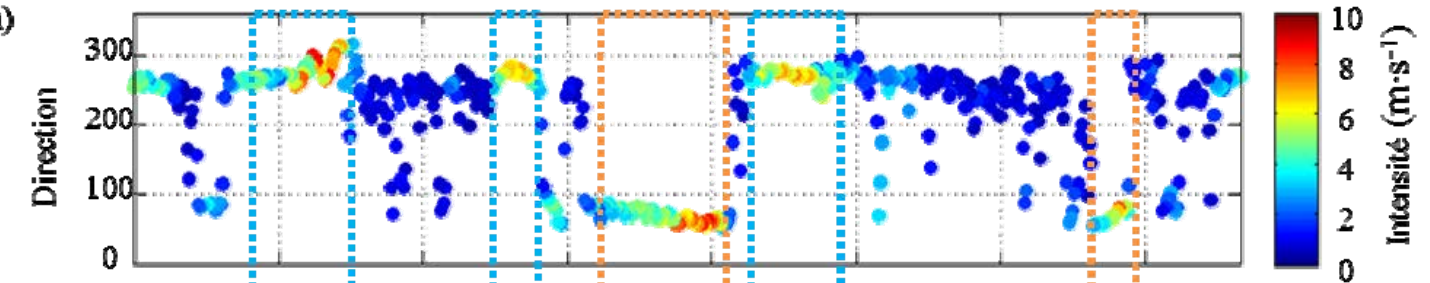

c)

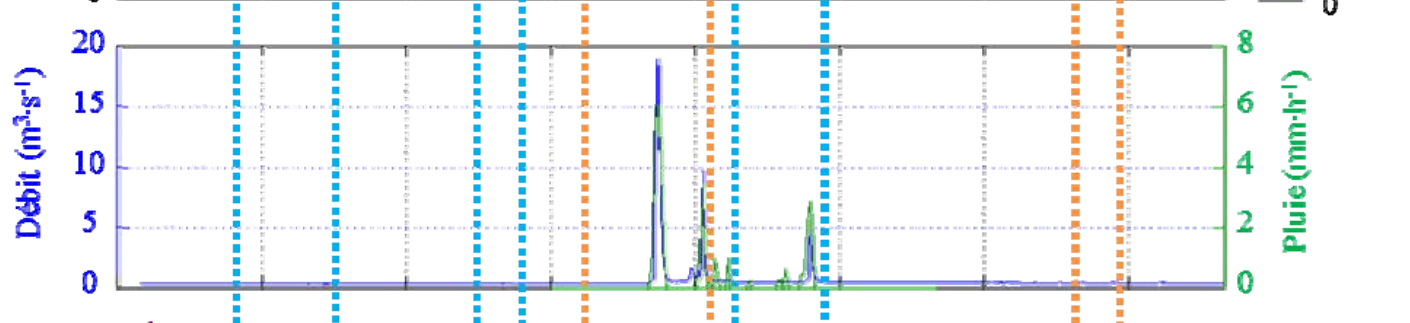

d)

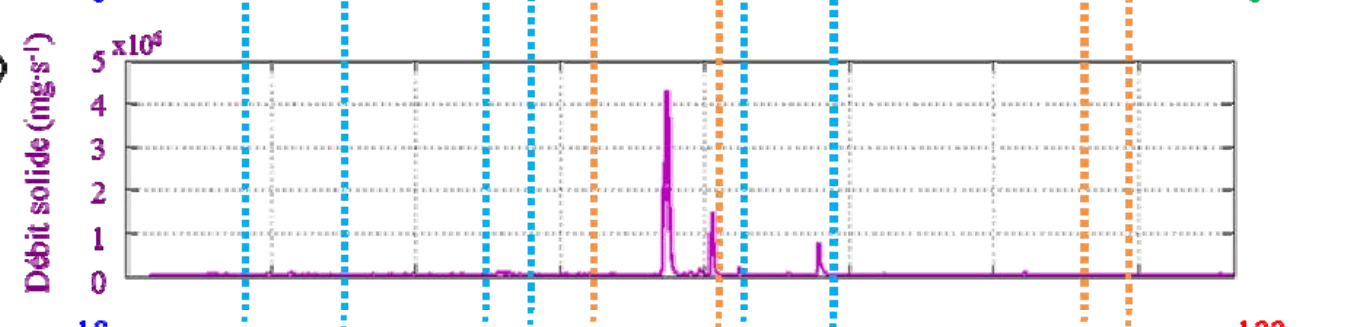

e)
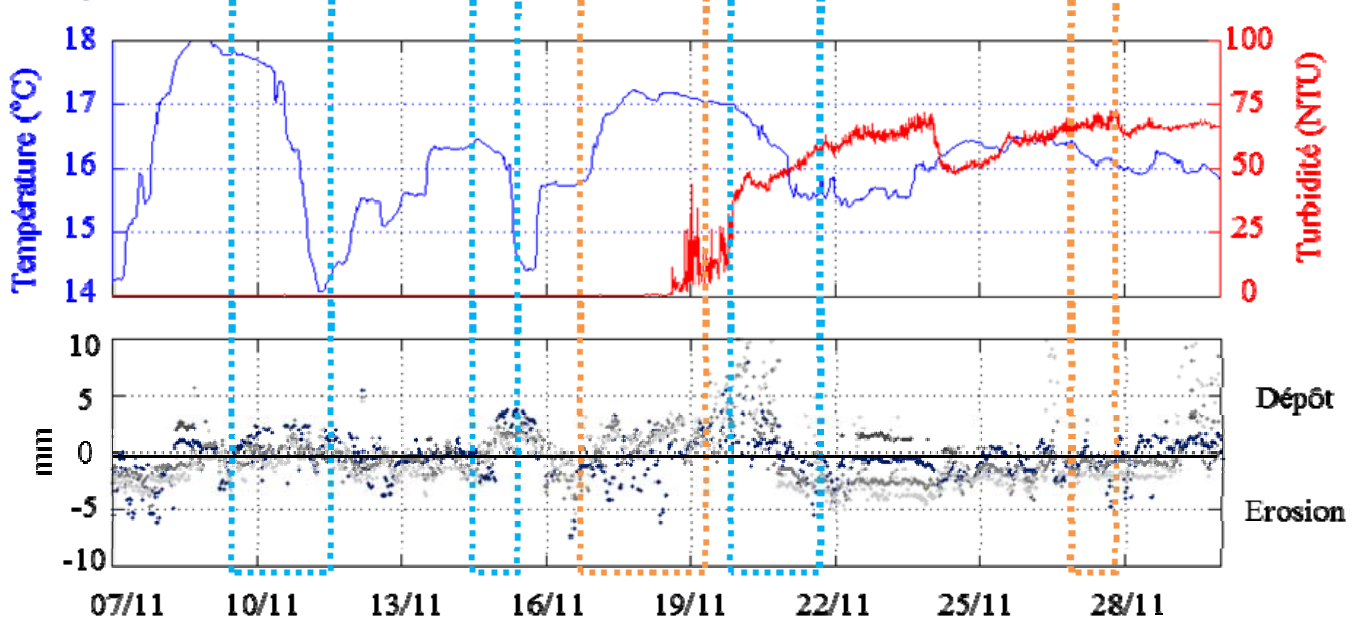

Figure 3. Evolution temporelle a) de l'intensité $\left(\mathrm{m} \cdot \mathrm{s}^{-1}\right)$ et direction du vent, b) du débit $\left(\mathrm{m}^{3} \cdot \mathrm{s}^{-1}\right)$ de l'Eygoutier et du cumul horaire de pluie, $\left.\mathrm{c}\right)$ du débit solide $\left.\left(\mathrm{mg} \cdot \mathrm{s}^{-1}\right), \mathrm{d}\right)$ de la température et turbidité (NTU) en mer et e) des mesures altimétriques par rapport au niveau initial pour les 4 voies de mesure, pour novembre 2013. Les épisodes de Mistral et Vent d'Est forts sont mis en évidence par les boites bleues et orange, respectivement.

\subsection{Dynamique sédimentaire}

Les données enregistrées en mer à proximité des exutoires montrent également une variabilité due aux épisodes pluvieux. Les apports de MES par les fleuves provoquent en effet une augmentation de la turbidité au fond, directement liée aux flux solides des fleuves (cf. épisodes de pluie 18, 19 novembre 2013 de la figure 3d). Très corrélés aux apports des fleuves, les pics de turbidité semblent beaucoup moins liés aux épisodes 


\section{Thème 2 - Dynamique sédimentaire}

ventés. En effet, les courants de fond sont plutôt faibles en fond de baie, où étaient mouillées les sondes, et ils n'apparaissent pas suffisants pour remettre en suspension le sédiment du bassin. Même lors d'épisodes de forts vents, c'est-à-dire dont l'intensité est supérieure à $5 \mathrm{~m} \cdot \mathrm{s}^{-1}$ (qui engendre l'apparition de vagues et moutons), aucune augmentation de l'opacité de l'eau n'est enregistrée. Les valeurs mesurées par temps calme sont d'ailleurs du même ordre de grandeur que celles par conditions ventées (5 à $10 \mathrm{mg} \cdot \mathrm{L}^{-1}$ ). Les résultats altimétriques montrent quant à eux de légères variations lors d'évènements fortement ventés. Les forts coups de vent génèrent de la remobilisation du sédiment de fond pour les deux sites étudiés. Les jours suivants un épisode de fort Mistral sont marqués par de la remise en suspension du sédiment, probablement à cause des fortes intensités près du fond du courant de décharge. De l'ordre de quelques millimètres, ces variations témoignent de la mobilité du sédiment, et pourraient justifier l'absence de pics de turbidité sur les données des sondes, fixées sur les cages ADCP à une trentaine de centimètres au-dessus du fond. Les apports de MES des fleuves par temps de pluie diminuent la distance altimètre/fond, signature d'un dépôt. Toutefois, les alternances de dépôt et remobilisation enregistrées ne mettent pas en évidence d'évolution du fond à plus long terme, qui malgré des variations d'ordre millimétrique, n'évolue pas sur la période étudiée. A titre d'exemple, la figure 3 illustre l'évolution temporelle des conditions de vent, des apports du fleuve Eygoutier et des mesures en mer à son exutoire pour le mois de novembre 2013.

\subsection{Modélisation hydro-sédimentaire}

Bien que les conditions ventées n'impactent pas beaucoup la turbidité au fond à proximité des exutoires, leur influence sur les panaches turbides en surface est plus marquée. En effet, le panache turbide sera dispersé vers le sud par Mistral et vers le nord-ouest par vent d'est. La figure 4 montre les concentrations de MES en surface simulées lors d'un épisode de vent d'Est. Bien qu'il reste encore à caler et valider le modèle avec des données in situ, les premières simulations (non présentées ici) montrent différentes zones de remobilisation, très dépendantes des conditions atmosphériques. Ces premiers résultats montrent une remise en suspension par Mistral des sédiments de la partie orientale de la Grande Rade ainsi que du Golfe de Giens. Par vent d'Est, la côte septentrionale de la Grande Rade et les côtes des Cap Cépet et Sicié sont impactées. Ces zones de fortes remises en suspension correspondent d'ailleurs aux régions où les proportions de sédiments fins sont les plus faibles.

\section{Conclusions et perspectives}

Principal moteur de la circulation, le vent impacte les directions et intensités du courant de la Rade de Toulon, en surface comme au fond. Bien que légèrement sous-estimés, les courants sont globalement bien reproduits par le modèle (DUFRESNE et al. 2014). Les inversions et renverses ainsi que les directions préférentielles semblent fidèles aux 


\section{XIII ${ }^{\text {èmes }}$ Journées Nationales Génie Côtier - Génie Civil \\ Dunkerque, 2-4 juillet 2014}

mesures in situ et le modèle reproduit bien la circulation dans la Rade. Les apports solides des deux fleuves se jetant dans la Rade ont été étudiés et leur signature turbide est visible aux deux exutoires. Les flux liquides et solides augmentent par temps de pluie et la majorité des sédiments apportés est composée de vase $(\mathrm{d}<63 \mu \mathrm{m})$. Les mesures en mer réalisées permettront désormais de caler les paramètres régissant la dynamique sédimentaire de la Rade. Ces données révèlent une alternance de dépôt et de remobilisation, sans cependant faire évoluer le fond par des érosions ou dépôts durables. Les mesures in situ permettront également de caler et valider le modèle hydrosédimentaire, notamment pour la dispersion des panaches turbides et les vitesses de chute.

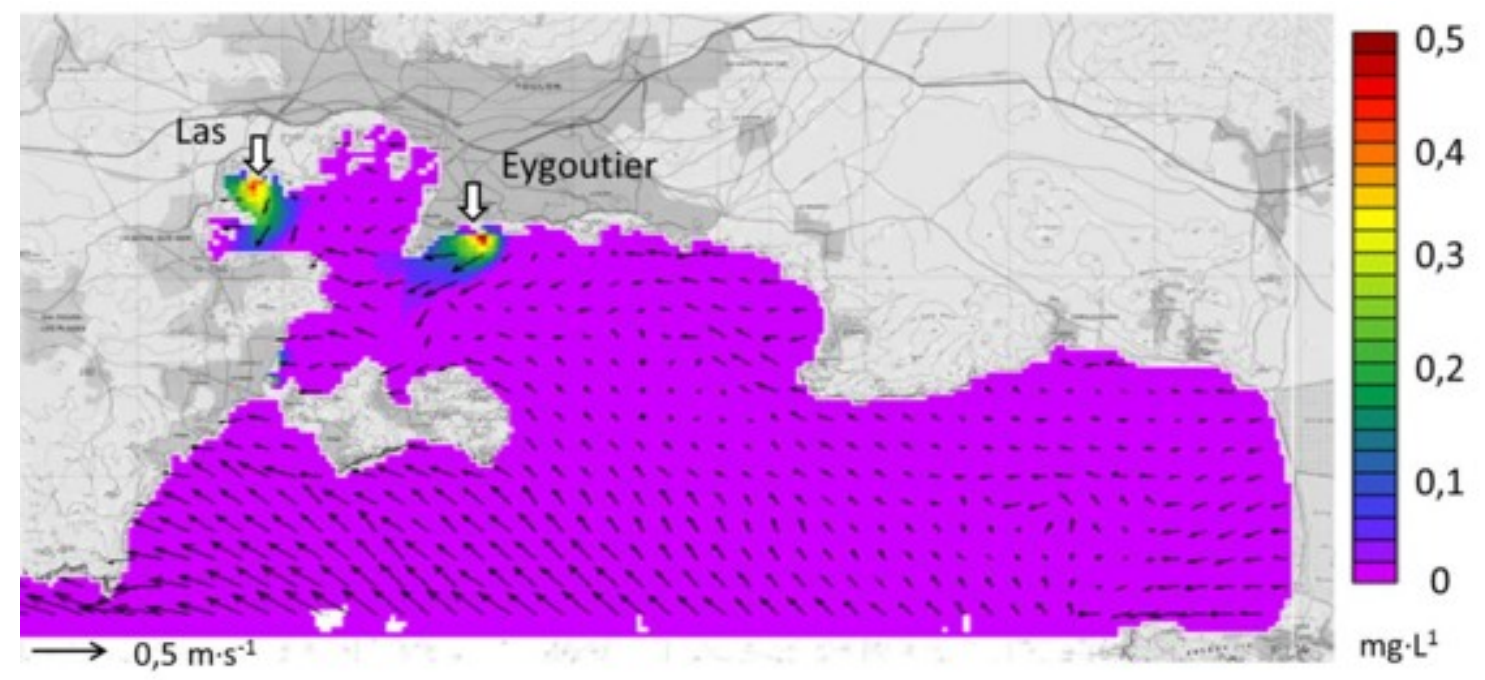

Figure 4. Concentrations de MES $\left(\mathrm{mg} \cdot \mathrm{L}^{-1}\right)$ modélisées (échelle couleurs) et courants de surface (flèches noires).

Ajoutés au modèle comme traceurs, les radionucléides pourront ensuite être pris en compte. Deux paramètres régiront leur dispersion : leur demi-vie ainsi qu'un coefficient de distribution (Kd) définissant leur proportion dissoute et particulaire. Les radionucléides adsorbés aux sédiments auront ainsi la même trajectoire (érosion, dépôt, transport). Différents scénarios pourraient être établis dans le but de minimiser l'impact d'une contamination en fonction des conditions atmosphériques et hydro-sédimentaires. Des simulations de dispersion de radionucléides pourraient fournir des éléments d'aide à la décision aux experts et décideurs en situation post-accidentelle.

\section{Références}

ARNAUD M., CHARMASSON S. (2000). Etude radioecologique de l'environnement marin de l'aire toulonnaise - Etude du compartiment sédimentaire. Campagne SIAT, IPSN/DPRE/SERNAT 2000-27. 
DUFFA C., DUFOIS F., COUDRAY S. (2011). An operational model to simulate postaccidental radionuclide transfers in Toulon marine area: preliminary development. Ocean Dynamics, Vol. 61(11), pp 1811-1821. http://dx.doi.org/10.1007/s10236-011-0429-0

DUFRESNE C., DUFFA C., REY V. (2014). Wind-forced circulation model and water exchanges through the channel in the Bay of Toulon. Ocean Dynamics, Vol. 64, pp 209-224. http://dx.doi.org/10.1007/s10236-013-0676-3

JOUAN M., GARREAU P., RAILLARD O. (2001). Elaboration d'une plate-forme de modelisation en Méditerranée: application à la Rade de Toulon. Rapport, Ifremer.

LAZURE P., DUMAS F. (2008). An external-internal mode coupling for a 3D hydrodynamical model for applications at regional scale (MARS). Advances in Water Resources, Vol. 31(2), pp 233-250. http://dx.doi.org/10.1016/j.advwatres.2007.06.010

LE HIR P., CAYOCCA F., WAELES B. (2011). Dynamics of sand and mud mixtures: A multiprocess-based modelling strategy. Continental Shelf Research, Vol. 31, pp S135-S149. http://dx.doi.org/10.1016/j.csr.2010.12.009

MILLOT C., BROYARD R., METAIS O., TINE J. (1981). Les oscillations propres de la rade de Toulon. Oceanologica Acta, Vol. 4(3), pp 259-262.

MONTE L., PERIAÑEZ R., BOYER P., SMITH J.T., BRITTAIN J.E. (2009). The role of physical processes controlling the behaviour of radionuclide contaminants in the aquatic environment: a review of state-of-the-art modelling approaches. Journal of Environmental Radioactivity, Vol. 100(9), pp 779-784. http://dx.doi.org/10.1016/j.jenvrad.2008.05.006 PHILLIPS J.M., WEBB B.W., WALLING D.E., LEEKS G.J.L. (1999). Estimating the suspended sediment loads of rivers in the LOIS study area using infrequent samples. Hydrol. Process, Vol. 13, pp 1035-1050. http://dx.doi.org/10.1002/(SICI)10991085(199905)13:7<1035::AID-HYP788>3.0.CO;2-K

PIROVANO G., COLL I., BEDOGNI M., ALESSANDRINI S., COSTA M.P., GABUSI V., LASRY F., MENUT L., VAUTARD R. (2007). On the influence of meteorological input on photochemical modelling of a severe episode over a coastal area. Atmospheric Environment Harbours and Air Quality, Vol. 41(30), pp 6445-6464. http://dx.doi.org/10.1016/j.atmosenv.2007.04.011

SCHAEFFER A. (2011). Impact du vent sur la circulation hydrodynamique dans le Golfe du Lion : modélisation haute résolution. Thèse Université Sud Toulon Var, 245 p. TESSIER E., GARNIER C., MULLOT J.-U., LENOBLE V., ARNAUD M., RAYNAUD M., MOUNIER S. (2011). Study of the spatial and historical distribution of sediment inorganic contamination in the Toulon bay (France). Marine Pollution Bulletin, Vol. 62(10), pp 2075-2086. http://dx.doi.org/10.1016/j.marpolbul.2011.07.022 